\title{
Ätiologie, Nomenklatur und Pathophysiologie der chronisch venösen Insuffizienz
}

\author{
W. Salmhofer
}

Eingegangen: 26. Februar 2016 / Angenommen: 13. Mai 2016 / Online publiziert: 14. Juni 2016

(C) The Author(s) 2016. This article is available at SpringerLink with Open Access

Zusammenfassung In diesem Artikel werden aktuelle Überlegungen zur Ätiopathogenese der primären Varikose und der chronischen venösen Insuffizienz präsentiert, die aktuelle Nomenklatur bzw. Terminologie von Venenerkrankungen, die kürzlich in einer internationalen Konsensuskonferenz festgelegt wurde, vorgestellt und die CEAP-Klassifizierung sowie das Venous Severity Score System besprochen.

Schlüsselwörter Ätiologie · Pathophysiologie · Nomenklatur · Venenerkrankungen · Chronische venöse Insuffizienz · CEAP · VSS-System

Etiology, nomenclature and pathophysiology of chronic venous insufficiency

Summary This article presents current notions and conceptions of the aetiopathogenesis of primary varicosis and chronic venous insufficiency, as well as an updated version of the nomenclature and terminology of venous disorders, which was recently agreed on in an international consensus conference. Furthermore, both CEAP-classification and venous severity score system are discussed.

Keywords Etiology · Pathophysiology · Nomenclature · Venous disease $\cdot$ Chronic venous insufficiency · CEAP . VSS-System

\section{Ätiologie der chronisch venösen Insuffizienz}

Die primäre Varikose ist eine degenerative Erkrankung der Venen des epifascialen Systems, die sich fast immer an den unteren Extremitäten manifestiert.

Die genaue Ursache für die Entstehung von Varizen ist noch nicht endgültig geklärt. Eine venöse Hypertension in den großen Venen mit primärer Klappeninsuffizienz und nachfolgender Dilatation der distalen Venenabschnitte ist nur in einem Teil der Fälle bereits zu Beginn nachzuweisen [1]. Nicht selten findet man - vor allem auch bei jüngeren Patienten - eine antegrade Entwicklung von Varizen mit pathologischen Refluxen und Ausweitungen von Venenwänden nur in Seitenästen, die Stammvenen sind (noch) nicht mitbetroffen [2-4].

Histologische Untersuchungen der Venenwand zeigten frühzeitig Veränderungen in der Intima, Tunica media und in der Adventitia, mit Vermehrung von Bindegewebs- und Muskelfasern sowie von extrazellulärer Matrix, Zerstörung von elastischen Fasern und Dysorganisation der Wandschichten mit nachfolgendem Re-,,Modelling“ der Venenwand [5]. Einwandern von Entzündungszellen, Freisetzen von Entzündungsmediatoren und Adhäsionsmolekülen, vermindertes Ansprechen auf vaso-konstriktorische Hormone und Hypoxie der Venenwand spielen in der Entstehung von Varizen eine Rolle [6].

Varizen können in jedem Lebensalter auftreten, die Prävalenz nimmt jedoch mit steigendem Alter zu. Begünstigende Faktoren für die Entstehung von Varizen sind zunehmendes Alter, familiäre Disposition, hormonelle Einflüsse, weibliches Geschlecht, Gravidität, Adipositas, stehender Beruf sowie vorangegangene Thrombose.

Im Laufe des Lebens kann es durch den Einfluss verschiedener Manifestationsfaktoren zur Dekompensation einer vorbestehenden Venenerkrankung kom- 
men und sich ein „Krampfaderleiden“ (chronisch venöse Insuffizienz, CVI) entwickeln, das durch ein Auftreten von trophischen Störungen bis hin zum Ulcus cruris venosum gekennzeichnet ist. Neben einer primären Varikose können auch Veränderungen des tiefen - subfaszialen - Venensystems (postthrombotische Veränderungen mit Obstruktion oder Insuffizienz der tiefen Leitvenen, Klappenagenesie u. ä.) zur Ausbildung einer CVI und manchmal auch von sekundären Varizen führen.

Eine rezente epidemiologische Untersuchung (Bonner Venenstudie) bestätigt die Häufigkeit sowohl der Varizen als auch der chronischen venösen Insuffizienz - weniger als $10 \%$ der erwachsenen Bevölkerung wiesen keinerlei Zeichen einer Varikose auf, während jeder sechste Mann und jede fünfte Frau von einer CVI betroffen waren. Hautveränderungen im Sinne einer fortgeschrittenen CVI fanden sich bei 3,4\% der Frauen und 3,8 \% der Männer [7]. In der FraminghamStudie lag die Zweijahres-Inzidenzrate für Varizen bei Männern bei 39,4/1000 Personen und bei Frauen bei $51,9 / 1000$.

\section{Nomenklatur bzw. Terminologie von Venener- krankungen}

In älteren Publikationen sind die Begriffe durchaus unterschiedlich bzw. uneinheitlich verwendet worden. Im Rahmen eines internationalen transatlantischen Konsensusdokuments wurde 2009 die Nomenklatur bzw. Terminologie für Venenerkrankungen überarbeitet und standardisiert [8]:

Chronisch venöse Veränderungen (chronic venous disorders). Umfaßt das volle Spektrum von morphologischen und funktionellen Auffälligkeiten des venösen Systems.

Chronisch venöse Erkrankung (chronic venous disease). Länger bestehende, morphologische und/ oder funktionelle Auffälligkeiten (z. B. Venenklappeninsuffizienz, sonographisch nachgewiesener pathologisch verlängerter Reflux) manifestieren sich mit Symptomen oder venösen Beschwerden, die eine weitere Abklärung bzw. Behandlung erforderlich machen.

Chronisch venöse Insuffzienz (CVI, chronic venous insufficiency, C3-C6). Dieser Begriff wird für funktionelle Abnormalitäten im venösen System reserviert, die zu trophischen Störungen der Haut (Ödeme, Pigmentierungen, Stasisdermatitis, Dermatosklerose, Atrophia alba) bis hin zu venösen Ulcera führen.

Venöse Symptome (venous symptoms). Beschwerden, die auf eine Erkrankung der Venen zurückzuführen sind (Brennen, Jucken, Schmerzen, Muskelkrämpfe, restless legs, schwere Beine, Juckreiz). Die Veränderungen sind NICHT pathognomonisch, kön- nen aber auf eine bestehende chronische venöse Erkrankung hinweisen.

Venöse Zeichen (venous signs). Sichtbare Manifestationen von venösen Veränderungen, inkl. Besenreiser- und retikuläre Varizen sowie große Varizen (Astund Stammvarizen), Beinödeme, trophische Hautveränderungen und venöse Ulcera (werden als klinische Zeichen $\mathrm{C}_{0}-\mathrm{C}_{6}$ im Rahmen der CEAP-Klassifikation erfasst).

Venöser Reflux (venous reflux). Retrograder venöser Fluss von abnormer Dauer in einem beliebigen venösen Segment - kann primär, sekundär oder congenital bedingt sein.

Segmentaler Reflux (segmental reflux). Lokalisierter retrograder Fluss in venösen Abschnitten des oberflächlichen bzw. des tiefen Systems oder im Perforantenbereich.

Axialer Reflux (axial reflux). Durchgehender retrograder venöser Fluss von der Leistenregion bis in die Wade (oberflächlich, tief, kombiniert möglich).

Rezidivvarizen (recurrent varices). Wiederauftreten von Varizen in einem vorher erfolgreich behandelten Areal.

Residualvarizen (residual varices). Varizen, die nach einer Behandlung von Varizen überbleiben.

PREVAIT. (Akronym für PREsence of Varices (residual or recurrent) After operatIve Treatment) fasst die Rezidiv- und Residualvarizen heute unter einem Begriff zusammen.

Postthrombotisches Syndrom (postthrombotic syndrome). Chronisch venöse Symptome und Zeichen als Folge eine vorangegangenen Venenthrombose.

Beckenvenensyndrom (pelvic congestion syndrome). Chronische Symptome, die auf eine Obstruktion oder einen Reflux im Bereich der Ovarialvene oder Beckenvene zurückzuführen sind. Das Beckenvenensyndrom kann sich durch Vulva-Varizen, perineale Varizen und/oder Varizen der Beine manifestieren und z. B. mit Beckenschmerzen, Schweregefühl, Harndrang und post-koitalen Schmerzen einhergehen.

Varicozele. Vorhandensein von skrotalen Varizen.

Venöses Aneruysma (venous aneurysm). Lokalisierte, sackförmige oder fusiforme Dilatation eines venösen Segments mit Durchmesserzunahme um mind. $50 \%$ gegenüber dem normalen Venendurchmesser.

Auch die Nomenklatur der oberflächlichen und tiefen Beinvenen wurde in den letzen Jahren diskutiert 
Tab. 1 Überblick über die anatomischen Bezeichnungen von Beinvenen

\begin{tabular}{|l|l|}
\hline Tiefes Venensystem der Beine & Oberflächliches Venensystem der Beine \\
\hline V. femoralis communis & $\begin{array}{l}\text { V. saphena magna } \\
\text { - Saphenofemorale Junktion } \\
\text { - Valvula terminalis } \\
- \text { Valvula präterminalis }\end{array}$ \\
\hline V. femoralis & V. pudenda externa \\
\hline V. femoralis profunda & V. circumflexa iliaca superficialis \\
\hline V. circumflexa femoris medialis & V. epigastrica superficialis \\
\hline V. circumflexa femoris lateralis & V. saphena accessoria anterior \\
\hline V. ischiadica & V. saphena accessoria posterior \\
\hline V. poplitea & V. saphena accessoria superficialis \\
\hline Plexus venosus genicularis & \\
\hline Vv. surales & V. saphena parva \\
\hline - Soleusvenen & - Sapheno-popliteal Junktion \\
\hline - Gastrocnemiusvenen & - Valvula terminalis \\
\hline V. tibiales anterior & - Valvula präterminalis \\
\hline V. tibialis posterior & Extensio cranialis venae saphenae parvae \\
\hline V. fibularis & V. saphena accessoria superficialis \\
\hline V. plantaris medialis & V. circumflexa femoris anterior \\
\hline V. plantaris lateralis & V. circumflexa femoris posterior \\
\hline Arcus plantaris profundus & V. intersaphena \\
\hline V. metatarsalis profundus (plantaris, dorsalis) & Plexus venosus dorsalis plantaris \\
\hline V. digitalis profundus (plantaris/dorsalis) & Arcus venosus dorsalis plantaris \\
\hline V. pedalis & V. metatarsalis superficialis (plantaris/dorsalis) \\
\hline & V. marginalis lateralis \\
\hline & V. marginalis medialis \\
\hline
\end{tabular}

und neu festgelegt (Tab. 1, [9, 10]). Hier sei darauf hingewiesen, dass die Bezeichnung „Vena femoralis superficialis“ zu vermeiden sei (richtig „V. femoralis“), und es wurden auch andere bisher gebräuchliche Namen ersetzt (z. B. statt V. saph. acc. lateralis und medialis werden nur die Namen „V. saph. acc. anterior“ bzw. „V. saph. acc. posterior“ verwendet). Eponyme sollen möglichst vermieden werden, als international akzeptiert gelten allerdings weiterhin die GiacominiAnastomose, die hintere Bogenvene, die Cockett-Perforantes und der Santorini-Plexus.

\section{Pathophysiologie der chronisch venösen Insuffi- zienz}

Um eine gezielte Behandlung der chronisch venösen Erkrankungen durchführen zu können, ist eine Kenntnis der Pathophysiologie von entscheidender Bedeutung.

Als Ausgangspunkt kommt es infolge angeborener und/oder erworbener Veränderungen der Venenwand oder einer Insuffizienz der Venenklappen - mit und ohne venöser Abflussstörung - zu einer venösen Hypertension in den Beinvenen, die sowohl die oberflächlichen subkutanen Venen, als auch das tiefe Leitvenensystem betreffen kann [11].

Es kommt - im Gegensatz zur physiologischen Situation - nicht mehr zu einem Druckabfall in den Venen beim Gehen, sondern zu einem Druckanstieg mit Druckspitzen bis zu 200 mm Hg. Dieser hohen
Drucke werden bis in die Mikrozirkulation wirksam und führen hier zu Veränderungen an den Arteriolen, Kapillaren und Venulen. In der Mikrozirkulation, die das Bindeglied zwischen venöser Hämodynamik und „kongestiven Dermatosen“ darstellt, kommt es zu vermehrter Durchlässigkeit der Kapillaren und verminderter Rückresorption von interstitieller Flüssigkeit im Bereich der Venulen; dies kann zunächst durch das Lymphgefäßsystem kompensiert werden. Bei längerem Bestand und zunehmender Schwere der venösen Schädigung kommt es dann aber zur Dekompensation des Systems, es entstehen venöse Ödeme.

Die venöse Hypertension führt in der Mikrozirkulation zur Dilatation, Elongation und Torquierung der Kapillaren und zu einer Verminderung des Blutflusses in nutritiven Kapillargefäßen. In weiterer Folge kommt es zum Austritt von Entzündungszellen in die Umgebung, Freisetzung von Entzündungsmediatoren (wie u. a. PAF, $\mathrm{LTA}_{4}, \mathrm{LTB}_{4}$ ) und Schädigung der Extrazellulärmatrix (Abb. 1) [12, 13].

Weiters kommt es zur Aktivierung von Neutrophilen und Thrombozyten in der Mikrozirkulation, die $\mathrm{zu}$ einer Aktivierung des Gerinnungssystems und zu einer Kontraktion der venulären Endothelzellen führen können [14]. Die chronische Entzündungsreaktion führt zu Fibrosierung und Sklerosierung, in Folge dann zum Umbau der Gefäßwand und Rarefizierung des nutritiven Gefäßnetzes sowie zum Abfall des transkutanen Sauerstoffpartialdrucks [15]. Dieser Prozess unterhält sich im Sinne eines „circulus vitious“ 
Abb. 1 Venöse Hypertension als hypothetische Ursache für die klinische Manifestation der CVI (adaptiert nach [13])

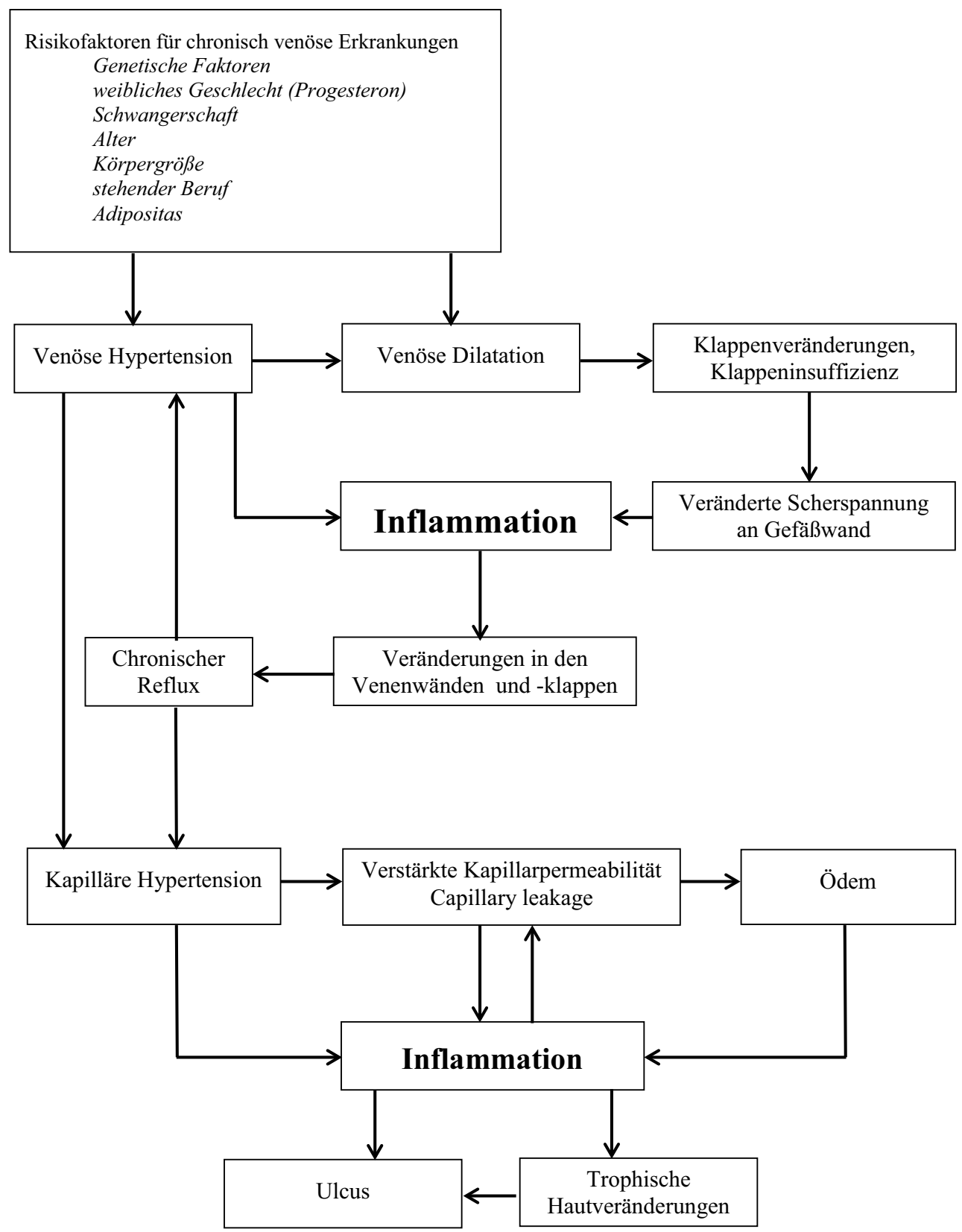

selbst. Als letztes - schwerstes - Studium kann sich schließlich ein Ulcus cruris venosum ausbilden. Infiltrate von Monozyten und Makrophagen finden sich aber auch in Gefäßwänden größerer Venen und insbesonders den Venenklappen, und führen hier zu einer weiteren Schädigung der Gefäßwand und Klappen in den großen Beinvenen.

\section{Klassifikation der chronischen venösen Insuffizi- enz}

Die Entwicklung einer chronisch venösen Insuffizienz erfolgt stufenweise über längere Zeit - meist dauert es Jahre, bis schwere Hautveränderungen auftreten. Die CVI kann sowohl bei primärer Varikose als auch bei Insuffizienz der tiefen Leitvenen beobachtet wer- den, und sich besonders bei Schädigung sowohl des oberflächlichen als auch tiefen Venensystems rascher entwickeln.

In der Routine wurde bisher in den deutschsprachigen Ländern meist die Widmer-Klassifikation verwendet. Sie erlaubt nur eine Beurteilung der chronisch venösen Insuffizienz (CVI) und führt die Klassifikation ausschließlich nach klinischer Symptomatik durch. Die Zuordnung im Rahmen der Widmer-Klassifikation ist sehr einfach durchzuführen, erfasst aber andere venöse Erkrankungen nicht (Tab. 2).

Eine Klassifizierung venöser Erkrankungen wird heute in wissenschaftlichen Publikationen nach der CEAP-Klassifikation vorgenommen $(\mathrm{C}=$ Clinical picture, $\mathrm{E}=$ Etiology, $\mathrm{A}=$ Anatomy, $\mathrm{P}=$ Pathophysiology) (Tab. 3; [16]). Mit der CEAP-Klassifikation können 
Tab. 2 Klassifikation der chronischen Veneninsuffizienz (CVI) nach Widmer

\begin{tabular}{|l|l|}
\hline Stadium & Klinische Symptome \\
\hline$I^{\circ}$ & Corona phlebectatica paraplantaris, venös bedingtes Ödem \\
\hline II $^{\circ}$ & $\begin{array}{l}\text { Ila: Ödem, Ekzem, Pigmentverschiebungen } \\
\text { Ilb: Dermatosklerose, Atrophie blanche }\end{array}$ \\
\hline III $^{\circ}$ & Stadium II + florides oder abgeheiltes Ulcus cruris \\
\hline
\end{tabular}

nicht nur die klinische Manifestationsform der venöse Erkrankung, sondern auch die individuellen Ursachen, die anatomische Lokalisation der Veränderungen und die wesentlichen pathophysiologischen Faktoren für den Patienten erfasst und klassifiziert werden; die CEAP-Klassifikation ist international anerkannt und ermöglicht eine generelle Klassifizierung von venösen Erkrankungen (chronic venous disorders), und nicht nur der CVI.

In der Routine wird meist die einfache CEAP-Klassifizierung (,basic CEAP“) verwendet. Neben der klinischen Beurteilung (C-Klassifikation - Angabe der höchsten C-Klasse) werden auch Daten zur Ätiologie, Anatomie und Pathophysiologie erhoben. Als Untersuchungsmethode der Wahl wird meist die farbcodierte Duplexsonographie (FCDS) verwendet.

Die „advanced CEAP-classification" ist eine noch detailliertere Klassifikation, die vor allem in Studien Verwendung findet. Hier werden die venösen Veränderungen umfassend erhoben (alle zutreffenden $\mathrm{C}$-Klassen werden notiert) und die pathophysiologische Veränderungen können 18 definierten Venensegmenten zugeordnet werden.

Die Klassifizierung nach CEAP ist nicht statisch, ein Patient kann jederzeit reklassifiziert werden; daher ist eine Datumsangabe der Untersuchung bzw. Klassifizierung sinnvoll. Auch die Wertigkeit der Untersuchung („Level of investigation“) sollte angegeben werden.

Level I: Anamnese, klinische Untersuchung incl. Handdoppler, ev. Farbdoppler-Untersuchung.

Level II: detaillierte nicht-invasive Untersuchungen, insbesonders Farbcodierte Duplexsonographie \pm Pletysmographie.
Level III: invasive bzw. aufwendige bildgebende Untersuchungen wie Phlebographie, Varikographie, venöse Druckmessungen, CT-Phlebographie und MRPhlebographie.

Als Ergänzung zur CEAP-Klassifizierung wurde ein Venous Severity Score-System (VSS-System) etabliert, um die Beschwerden des Patienten besser zu erfassen und ein Instrument zur Verfügung zu haben, um Behandlungsergebnisse zu bewerten. Das VSS-System erfasst sowohl venöse Symptome als auch venöse Zeichen, und ist geeignet, den klinischen Outcome in Studien zu erfassen und zu evaluieren [17].

Das VSS-System umfasst 3 Komponenten:

\section{Venous Disability Score (VDS)}

0 = asymptomatisch.

1 = symptomatisch, kann aber alle üblichen Aktivitäten ohne Kompressionstherapie ausüben.

2 = symptomatisch, kann die üblichen Aktivitäten nur mit Kompressionstherapie und/oder Hochlagerung der Extremitäten ausüben.

3 = symptomatisch, kann auch mit Kompression und/ oder Hochlagerung der Extremitäten die üblichen Aktivitäten nicht mehr ausüben.

\section{Venous Segmental Disease Score (VSDS)}

Kombiniert anatomische und pathophysiologische Komponenten aus der CEAP-Klassifikation für bestimmte Venenabschnitte. Die Bewertung basiert gänzlich auf bildgebende Untersuchungsmethoden (FCDS, Venographie ...). Es wird ein Wert (Score) für den Reflux (0-10) erfasst, und ein Score für Obstruktion (0-10).

\section{Venous Clincial Severity Score (VCSS)}

Neun klinische Charakteristika (Schmerzen, variköse Venen, venöses Ödem, Hautpigmentierung, Inflammation, Induration, sowie Zahl, Dauer und Größe aktiver Ulcera) werden erfasst und bewertet (0-3 Punkte), und auch die zusätzliche Verwendung konservativer Therapiemaßnahmen (Kompression, Elevation)

Tab. 3 Basic CEAP-Klassifikation

\begin{tabular}{|c|c|c|c|}
\hline $\begin{array}{l}\text { Klinsches Bild } \\
\text { Clinical Picture }\end{array}$ & $\begin{array}{l}\text { Ätiologie } \\
\text { Etiology }\end{array}$ & $\begin{array}{l}\text { Anatomie } \\
\text { Anatomy }\end{array}$ & $\begin{array}{l}\text { Pathophysiologie } \\
\text { Pathophysiology }\end{array}$ \\
\hline $\begin{array}{l}\mathrm{C} 0=\text { keine Varizen } \\
\mathrm{C} 1=\text { Teleangiektasien, reticuläre Varizen } \\
\mathrm{C} 2=\text { Ast- und Stammvarizen } \\
\mathrm{C} 3=\text { Ödeme } \\
\mathrm{C} 4=\text { Hautveränderungen } \\
\mathrm{C} 4 \mathrm{a}=\text { Ekzem, Hyperpigmentierung } \\
\mathrm{C} \mathrm{b}=\text { Lipodermatosklerose, Atrophie blanche } \\
\mathrm{C} 5=\text { abgeheiltes Ulcus } \\
\mathrm{C} 6=\text { florides Ulcus }\end{array}$ & $\begin{array}{l}E_{\mathrm{P}}=\text { primär } \\
E_{\mathrm{S}}=\text { sekundär } \\
E_{\mathrm{C}}=\text { kongenital } \\
E_{\mathrm{N}}=\text { keine Anomalie }\end{array}$ & $\begin{array}{l}A_{S}=\text { superfizieller Reflux } \\
A_{D}=\text { tiefer Reflux } \\
A_{P}=\text { Reflux aus Perforantes }\end{array}$ & $\begin{array}{l}P_{R}=\text { Reflux } \\
P_{0}=\text { Obstruktion } \\
P_{R, 0}=\text { Reflux und Obstruktion }\end{array}$ \\
\hline
\end{tabular}


mit 0-3 Punkten bewertet. Es kann beim VCSS ein Punktemaximum von 30 Punkten erreicht werden.

Das VSS-System wurde in mehreren Studien validiert, die Werte korrelieren gut mit dem Schweregrad der venösen Erkrankung. Der VCSS ist ähnlich sensitiv wie die CEAP-Klassifikation und besser geeignet, Änderungen des klinischen Schweregrade nach therapeutischen Maßnahmen - wie z. B. nach Operationen - zu erfassen. Auch für die globale Abschätzung des Schweregrades der chronisch venösen Erkrankung ist der VCSS besser als die CEAP-Klassifikation [18, 19].

Interessenkonflikt W. Salmhofer gibt an, dass kein Interessenkonflikt besteht.

Open Access This article is distributed under the terms of the Creative Commons Attribution 4.0 International License (http://creativecommons.org/licenses/by/4.0/), which permits unrestricted use, distribution, and reproduction in any medium, provided you give appropriate credit to the original author(s) and the source, provide a link to the Creative Commons license, and indicate if changes were made.

\section{Literatur}

1. Trendelenburg F. Über die Unterbindung der Vena saphena magna bei Unterschenkel-varicen. Beitr Klin Chir. 1890; 7:195-210.

2. Caggiati A, Rosi C, Heyn R, et al. Age-related variations of varicose veins anatomy. JVasc Surg. 2006;44:1291-5.

3. Dodd H. Varicous tributaries of the popliteal vein. Br J Surg. 1965;52:350-4.

4. Golledge J, QuigleyFG. Pathogenesis of Varicose Veins. Eur J Vasc Enodvasc Surg. 2003;25:319-24.

5. Leu HJ. Morphology of chronic venous insufficiency - light and electron microscopic examinations. Vasa. 1991;20:330-42.

6. Segiet OA, Brzozowa-Zasada M, Piecuch A, et al. Biomolecular Mechanisms in Varicous Vein Development. Ann Vasc Surg. 2015;29:377-84.

7. Rabe E, Pannier-Fischer F, Bromen K, et al. Bonner Venenstudie der Deutschen Gesellschaft für Phlebologie.
Epidemiologische Untersuchung zur Frage der Häufigkeit und Ausprägung von chronischen Venenkrankheiten in der städtischen und ländlichen Wohnbevölkerung. Phlebologie. 2003;32:1-14.

8. Eklöf B, Perrin M, Delis KT, et al. Updated terminology of chronic venous disorders: The VEIN-TERM transatlantic interdisciplinary consensus document. J Vasc Surg. 2009;49:498-501.

9. Caggiati A, Bergan JJ, Gloviczki P, et al. Nomenclature of the veins of the lower limbs: an international interdisciplinaryconsensus statement. JVasc Surg. 2002;36:416-22.

10. Caggiati A, Bergan JJ, Gloviczki P, et al. Nomenclature of the veins of the lower limb: Extensions, refinements, and clinical application. J Vasc Surg. 2005;41:719-24.

11. Eberhardt RT, Raffetto JD. Chronic Venous Insufficiency. Circulation. 2005;111:2398-409.

12. Nicolaides A, Kakkos S, Eklof B, et al. Pathophysiology in Management of chronic venous disorders of the lower limbs: guidelines according to scientific evidence. Int Angiol. 2014;33:88-94.

13. Bergan JJ, Schmid-Schönbein GW, Coleridge SPD, et al. Chronic Venous Disease. NEngl J Med. 2006;355:488-98.

14. Nees S, Juchem G, Weiss DR, Partsch H. Pathogenese und Therapie der Chronischen Venenerkrankung. Phlebologie. 2012;41:246-57.

15. Nicolaides AN. Investigations of Chronic Venous Insufficiency. Circulation. 2000;102:e126:e163.

16. Eklöf B, Rutherford RB, Bergan JJ, et al. American Venous Forum International Ad Hoc Committee for Revision of the CEAP Classification. Revision of the CEAP classification for chronic venous disorders: consensus statement. J Vasc Surg. 2004;40:1248-52.

17. Nicolaides A, Kakkos S, Eklof B, et al. Classification, severity scoring systems and terminology of chronic venous disorders in Management of chronic venous disorders of the lower limbs: guidelines according to scientific evidence. Int Angiol. 2014;33:104-10.

18. Nicolaides A, Kakkos S, Eklof B, et al. Investigations in Management of chronic venous disorders of the lower limbs: guidelines according to scientific evidence. Int Angiol. 2014;33:111-4.

19. Kakkos SK, Rivera MA, Matsagas MI, et al. Validation of the newvenous severity scoring system in varicose vein surgery. JVasc Surg. 2003;38:224-8. 